regretted that teacher training has not developed. An examen de maturité following the secondary school leaving examination has been established. Of late, technical education has seen some important transformations: Association of technical and agricultural education to the National Ministry of Education, regularisation of structures and conditions for nomination of teachers, unification of programmes, evaluation of agricultural education. The reform of art education remains to be undertaken. In secondary, as in primary education, the application of increasingly active methods is recommended: language laboratories, individual assignments, discussion of new mathematics, "positive aesthetics" (esthétique positive) etc. Belgium is abandoning more and more its tradition of autonomous, secondary level Ecoles normales in order to train teachers at the level of higher, but non-university, education. After a study of the humanities, future teachers will devote a year to professional training. Considering this preparation insufficient, the creation of teacher training institutes (Instituts de formation pédagogique) at a level more advanced than the secondary has been recommended in certain circles.

The charges mentioned are less the result of real reform plans (plans de réforme) than of a series of individual measures ordered by circumstances.

\title{
LA PEDAGOGIE EXPERIMENTALE EN BELGIQUE
}

Malgré des moyens humains et matériels fort limités, la Belgique est un lieu d'élection de la pédagogie expérimentale. Depuis plusieurs décennies, quelques chercheurs de haute qualification tels que $R$. Buyse et $F$. Hotyat font le renom international du pays. Grâce à eux, la Belgique a pu emboîter le pas aux pays anglo-saxons qui, depuis 1945, exercent un leadership indiscutable et amorcer un mouvement dont on ne doit pas sous-estimer l'importance. Le terrain est prêt pour une expansion spectaculaire - le développement rapide du nouveau Laboratoire de Pédagogie expérimentale de l'Université de Liège en est un exemple -; il suffirait de quelques millions de francs (le pays n'a ni Ford Foundation, ni Volkswagenstifiung) et de quelques dizaines de chercheurs supplémentaires.

Les notes qui suivent se rapportent essentiellement à la pédagogie expérimentale; elles ne couvrent donc pas les recherches théoriques relatives à la philosophie, l'histoire, la pédagogie générale, les méthodologies, la statistique, etc.; les travaux de psychologie sont aussi en dehors de notre propos.

Le peu de place qui nous est imparti nous a contraint à échantillonner arbitrairement parmi les recherches en cours et à réduire fortement la bibliographie. Le plus souvent, nous décrivons l'activité des centres de recherches plutôt que celle des individus qui s'y rattachent ou même en font partie de façon permanente. Comme il s'agit de recherches récentes, voire projetées, nous avons évidemment été limités par les informations qui nous ont été communiquées.

Les grands organismes belges s'intéressant exclusivement ou en partie à la pédagogie expérimentale sont les suivants:

- Laboratoire de Pédagogie expérimentale de l'Université de l'Etat à Liège, Dir. Prof. G. de Landsheere (Liège) ${ }^{1}$ ),

- Laboratoire de Pédagogie expérimentale de l'Université de l'Etat à Gand, Dir. Prof. R. Verbist (Gand),

1) Dans la suite de ce texte, les mentions entre parenthèses serviront à désigner les organismes cités. 
- Centre de Sociologie de l'Education de l'Université Libre de Bruxelles, Dir. Prof. S. de Coster (Bruxelles),

- Laboratoire de Pédagogie expérimentale de l'Université Catholique de Louvain, section française: Dir. Prof. A. Gille (Louvain, F.) I), section néerlandaise: Dir. Prof. V. d'Espallier (Louvain, N.) ${ }^{2}$ ),

- Institut Supérieur de Pédagogie du Hainaut, repris par le Centre Universitaire de l'Etat, à Mons, Dir. F. Hotyat (I.S.P.H.),

- Centre National de Recherches de Psychotechnique Scolaire, Président: R. Buyse (C.N.R.P.S.),

- Direction des Centres Psycho-Médico-Sociaux de 1'Etat, Dir. R. Derivière (P.M.S.),

- Laboratoire de Psychologie appliquée et organismes y attachés. Dir. Prof. L. Coetsier (Gand L.P.A.),

- Service de Psychologie génétique, Dir. Prof. A. Kriekemans (Louvain S.P.G.),

- Bureau pédagogique du Secrétariat National de l'Enseignement catholique, Dir. Chan. M. Nauwelaerts - Sécrétaire général: Abbé R. Beirnaert (E.C.),

- Commission Consultative Universitaire de Pédagogie (organisme d'Etat comprenant un secrétariat permanent et une représentation des quatre Universités) (C.C.U.P.).

Avant de synthétiser les différentes recherches spécifiques, on signale d'abord la publication de quelques ouvrages généraux destinés à tracer les cađres théoriques et à fournir les outils de base.

En 1964, G. de Landsheere publie son Introduction à la recherche pédagogique(1) ouvrage proposant une première taxonomie de la recherche éducationnelle et apportant une analyse critique des méthodes, des techniques et des instruments. Le même auteur publie en 1965, Les tests de connaissances(2) bilan des épreuves objectives existant en langue française et description des principaux tests étrangers. Outre son rôle d'initiateur, ce second ouvrage tente d'établir un état des besoins et a d'ailleurs suscité immédiatement la mise en chantier de plusieurs épreuves.

Dans la même ligne, il faut citer l'ouvrage de $\mathrm{H}$. Rigaux où l'élaboration d'un test de connaissances est décrite étape par étape(3), l'étude sur la validation de $P$. Frankart(4) et la traduction de l'ouvrage désormais classique que Davis a consacré à l'analyse des items(5).

\section{Sociologie de l'éducation}

L'aspect sociologique des problèmes éducatifs apparaît aujourd'hui d'une telle importance que l'on voit se tourner à la fois vers lui des sociologues, des psychologues et des pédagogues.

Le secteur sociologique est illustré par deux enquêtes de P. Minon et de l'équipe de l'Institut de Sociologie de Liège, l'une sur l'enseignement secondaire dans la région liégeoise (6), l'autre sur les facteurs sociaux de la première orientation scolaire (7), importante publication qui met cruellement en lumière le handicap éducatif croissant des enfants issus des familles laborieuses.

En psycho-sociologie, on note les recherches de L. Coetsier et al. qui mettent en lumière la position d'infériorité de la jeunesse flamande dans les secteurs de l'enseignement qui répondent le mieux aux besoins de la civilisation contemporaine (8) (9).

1) $\mathrm{F}$. = Français.

2) N. = Néerlandais. 
A partir de données concernant le degré de scolarisation et les résultats de l'examen psychologique de miliciens belges, des cartogrammes du potentiel intellectuel disponible dans chaque région du pays ainsi que son niveau d'exploitation par les différentes formes de scolarisation ont été dressés (10).

Dans le secteur pédagogique, la collaboration de F. Hotyat et de S. de Coster (Centre de Sociologie de l'Education de l'U.L.B.) nous vaut plusieurs publications sur la régression sociale et sur la relation entre les conditions socio-culturelles de la famille et le rendement scolaire(11) (12).

Partant de ce dernier travail, des moyens de combattre la détériorisation progressive des résultats scolaires sont essayés dans une classe expérimentale de jeunes filles (début de l'enseignement commercial) (I.S.P.H.).

A Louvain (F.), on note une étude socio-psychologique des futures institutrices gardiennes et une recherche sur la relation entre le statut sociométrique des élèves et l'opinion des professeurs. L'évolution du statut sociométrique dans des classes de neige a été mesurée à Liège (13).

\section{Méthodologie générale}

En raison de sa valeur exceptionnelle, on signale l'ouvrage théorique d'A. Clausse (Liège) sur la philosophie de l'étude du milieu (14).

En partie selon des méthodes expérimentales, G. de Landsheere a tenté de définir une pédagogie de la divergence(15).

Une méthodologie mésologique est élaborée à Louvain (F.).

\section{Programmes}

Pour les réseaux scolaires officiel et catholique, des réformes des programmes de l'enseignement secondaire général ou technique et des universités sont en cours; souvent, elles sont introduites sans appui direct sur la recherche.

La charnière du primaire et du secondaire fait exception. La direction générale de l'organisation des études, du Ministère de l'Education, en collaboration avec les centres P.M.S., a lancé, en 1966, une recherche pour définir une pédagogie de transition au niveau de la première année du secondaire. Les élèves de 42 écoles ont été testés en langue maternelle et en calcul; des groupes de rattrapage avec un enseignement programmé sont constitués. Pour les résultats se situant sous la moyenne, des examens approfondis sont entrepris et un rattrapage culturel est organisé.

L'élaboration et l'évaluation d'un programme d'enseignement correctif pour élèves peu doués, de 12 à 13 ans, est aussi en cours à Louvain (F.).

Pour les élèves inaptes à suivre un enseignement secondaire du niveau fort et qui manquent d'intérêt pour l'enseignement professionnel existant, 1'E.C., en collaboration avec Louvain (N.), expérimente dans dix écoles un "enseignement professionnel polyvalent" (formation générale adaptée et habileté multilatérale).

Une enquête relative aux programmes de langue maternelle (français et néerlandais) a été réalisée par la C.C.U.P.

\section{Méthodologie spéciale et programme des branches}

En langue maternelle, on note une recherche sur les méthodes d'acquisition de la lecture (C.C.U.P.), l'élaboration d'une méthode d'apprentissage de l'orthographe et une étude sur la difficulté des textes lus à l'école primaire (Louvain, F.), point qui est aussi traité dans une recherche lancée par Liège. 
En mathématiques, Louvain (F.) et C.C.U.P. prennent une place importante: aptitude spatiale dans l'enseignement gardien - sujet également étudié à Liège -; pour l'école primaire: mathématique moderne, arithmétique(16), formes géométriques (17), division écrite des nombres entiers(18); pour le secondaire: mathématique moderne.

A Louvain (F.), on note encore des recherches sur l'enseignement de la morale chrétienne et des sciences (niveau secondaire), sur l'éducation civique, sur la méthode Ramain et sur le dessin (Louvain, S.P.G.).

Gand se distingue par une recherche importante sur la formation plastique (nouvelle méthodologie de l'enseignement du dessin) (19) et par une étude sur l'aptitude musicale (patrimoine tonal de l'enfant, psychologie du chant autonome, expression musicale de l'enfant) (20). Gand s'attache aussi à la méthodologie de l'enseignement de la seconde langue.

Evaluation objective, mesure des connaissances, études de rendement, tests.

a) Recherche fondamentale

S'appuyant sur l'équipement de son Centre Interfacultaire de Calcul (Ordinateur I.B.M. 7040 et Gamma Bull), Liège poursuit depuis plusieurs années une vaste recherche sur la lisibilité (readability) et sur la lecture. L'Indice Flesch-de Landsheere a été provisoirement adopté pour le français (21) (22); ont été rejetés: la méthode courte et le score d'intérêt humain de Flesch, ainsi qu'un indice (Liège) basé sur le pouvoir séparateur de la ponctuation (23).

Le vocabulaire des manuels scolaires et des périodiques est évalué par des indices, l'un fondé sur une pondération basée sur les fréquences, l'autre sur le vocabulaire du Français Fondamental. Des séries de manuels de langue maternelle et d'histoire sont étudiées, de même que quatre périodiques pour enfants et deux quotidiens pour adultes (Liège).

Liège entreprend actuellement une recherche sur la capacité en lecture, à partir d'analyses ophtalmographiques.

La C.C.U.P. a lancé plusieurs recherches originales sur la façon dont les élèves de 6e primaire de différents cantons flamands parlent le néerlandais (24).

Le vocabulaire des élèves de l'enseignement secondaire a été étudié à Liège (C.N.R.P.S.) (25).

\section{b) Aptitudes et maturité pédagogique}

L'importance de la recherche de V. d'Espallier sur la maturité scolaire (spécialement maturité en lecture et en calcul) reste grande(26). Une étude longitudinale de la maturité scolaire est aussi entreprise par la C.C.U.P. M. Maquet a expérimenté le Frankfurter Schulreifetest sur une population liégeoise (Liège).

On doit à L. Coetsier et à ses collaborateurs une batterie différentielle d'aptitudes pour la charnière primaire-secondaire (27) (28). Des épreuves pour la transition secondaire inférieur-secondaire supérieur et secondaire supérieur-université sont en voie d'élaboration.

Une épreuve d'aptitudes pour le début du secondaire, portant sur les facteurs $\mathrm{v}, \mathrm{s}, \mathrm{n}, \mathrm{r}$, est en construction au C.N.R.P.S.

Portant sur 1.500 élèves de 61 établissements et validée par un follow up de quatre ans, une batterie comprenant des tests mentaux (Bonnardel), une épreuve 
d'intérêts (Derivière) et deux tests de connaissances (Arbalestrie) permet la différenciation des aptitudes littéraires ou scientifiques (P.M.S.).

K. Swinnen, enfin, a élaboré une batterie destinée à prédire le succès dans les. Humanités (Humanoriatest, 1957).

\section{c) Rendement et diagnostic}

- Lecture: J. Burion (I.S.P.H.) étudie actuellement les variations du rendement des élèves des premières années primaires, selon les méthodes d'apprentissage qu'ils ont suivies. Aux tests de lecture relativement récents (29) vont s'en ajouter plusieurs: adaptation des California Reading Tests pour le primaire et le secondaire (Louvain, F.), lecture silencieuse en 6e primaire (Gand), tests pour les niveaux 12-15-18 ans (Liège).

- Langue maternelle. Tests construits ou en cours de construction: G. Pire(Liège) (30), analyse, conjugaison, orthographe, lecture silencieuce (A. Bonboir, Louvain, F.); la rédaction française est étudiée par Delépine (I.S.P.H.) et par l'E.C. (néerlandais), en collaboration avec l'Institut de linguistique appliquée de Louvain (N.).

- Mathématiques. Arithmétique et formes géométriques: épreuves aux divers niveaux du primaire, étiologie des erreurs, traitement individualisé pour les fractions, la numération décimale, les formes géométriques: A. Bonboir (Louvain, F., C.C.U.P.) (31) (32); arithmétique et formes géométriques: (Wathelet) (34); R. Dujardin et L. Adriaenssens (33); arithmétique fin du primaire: G. Pire (35), calcul, première et deuxième du primaire: $L$. Cleempoel et $F$. Hotyat, arithmétique $5 \mathrm{e}$ primaire H. Rigaux(3); épreuve analytique d'arithmétique, fin du primaire: A. Bongrain et al. (I.S.P.H.); tests de mathématiques, fin du premier cycle secondaire et pré-universitaire pour candidats boursiers africains (I.S.P.H.).

Une enquête sur les connaissances en arithmétique, à la fin du primaire, dans des écoles expérimentales flamandes est en cours (C.C.U.P.).

- Langues: latin: Pire (36) et Louvain (F.); allemand: Louvain (F.).

- Géographie (Vocabulaire à l'école primaire): L. Dufrenne (Louvain, F. - C.C.U.P.).

- Histoire: fin du primaire: R. Verbist (Gand).

- Sciences économiques dans le secondaire supérieur (Liège).

- Tests portant sur plusieurs branches. Les tests E.P. 6 (fin primaire): A. Van Wayenberghe(37); inventaire de connaissances en fin du secondaire inférieur: Arbalestrie (38); du secondaire supérieur (C.N.R.P.S.) (39).

- Rendement des études supérieures: supériorité des étudiants boursiers: L. Coet$\operatorname{sier}(40)(41)$.

- Rendement des classes de neige, rendement en arithmétique et en orthographe, et évolution du statut socio-économique (Liège); rendement général (E.C.).

\section{Bilinguisme}

Gand étudie l'incidence du bilinguisme sur le développement intellectuel; Louvain (N.), et spécialement V. d'Espalier, s'attachent au même problème.

\section{Enseignement programmé}

Un prototype de machines, "DOCEO I," pour programmes polyséquentiels avec exploitation permanente par ordinateur a été construit à Liège (Centre de Calcul). Des recherches psychopédagogiques de programmation sont en cours à Louvain (F.) et à Liège. La theorie de la programmation polyséquentielle fait l'objet de nouveaux développements (Liège - Centre de Calcul). Des programmes sont en construction 
à Liège (participe passé, 15 ans; fractions et pourcentages, 6e primaire), à l'I.S.P.H. (carnets de réapprentissage: orthographe, grammaire) et à Louvain (F.).

\section{Audio-visuel}

Sur le plan général, on peut signaler deux enquêtes de la C.C.U.P.: Les auxiliaires audio-visuels au service de l'enseignement primaire et l'utilisation des audio-visuels pour l'enseignement de la seconde langue.

On note plusieurs recherches relatives à la Télévision: Télévision scolaire et apprentissage des sciences (Louvain, F.), influence de la T.V. sur la vie familiale (Louvain, S.P.G.), prétesting des émissions de T.V. pour enfants (Liège), enseignement et formation des maîtres par T.V. en circuit fermé (Liège), vocabulaire et difficulté de compréhension de leçons par T.V. scolaire (Liège), mise au point d'un indice de visibilité (Liège).

Une méthode d'initiation cinématographique est expérimentée à Louvain (F.). L'emploi du disque au jardin d'enfants et en première primaire est étudié à Louvain (F.).

\section{Attitudes}

La méthodologie de la construction des échelles d'attitudes à fait l'objet d'importants travaux à Liège (45).

Plusieurs échelles ont été construites : attitudes envers les mathématiques (Gand; Liège), envers Dieu, l'armée et le service militaire, les personnes âgées; attitudes des professeurs envers les élèves, des parents envers les mouvements de jeunesse (Louvain, F. et N.).

\section{Docimologie}

Sur le plan théorique, on note l'ouvrage de F. Hotyat(42) et une mise au point générale en cours à Liège.

Aspects particuliers: analyse des examens cantonaux (C.C.U.P.-I.S.P.H.), spécialement des résultats aux épreuves orthographiques 1964, en lecture et en conjugaison (C.C.U.P.-I.S.P.H.), comparaison entre le résultat aux examens ordinaires et aux tests de français et d'arithmétique (C.C.U.P.), les examens d'entrée dans l'enseignement moyen (C.N.R.P.S.), les tâches après la classe F. Hotyat (43), la signification de l'examen de maturité (Gand).

\section{Dossiers, guidance et orientation scolaires}

Une fiche scolaire longitudinale de F. Hotyat et Manouvrier sera publiée en cette année.

Les P.M.S. mettent au point un système pour la prévention de l'inadaptation en première primaire et la guidance des inadaptés de l'enseignement primaire.

Autres problèmes traités: facteurs influençant le choix des études dans la province de Luxembourg (Louvain, F.) dépistage et diagnostic des élèves normalement doués présentant des difficultés d'apprentissage (Louvain, N.), les retardés pédagogiques dans les écoles d'enseignement général (C.C.U.P.).

\section{Teaching}

Une importante recherche (1re tranche: 10 ans) est en cours à Liège: analyse des fonctions de l'enseignement, mise au point d'un schéma d'analyse de contenu, recherche pilote en première année primaire, recherche sur les patterns de fonctions. 
On note aussi des études sur quelques schèmes de comportements d'enseignants (Gand) et sur l'absence de critères d'orientation des futurs maîtres (Louvain, F.).

\section{Handicapés}

Le plus grand nombre de recherches signalées concerne les sourds : raisonnement dans la solution de problèmes chez les sourds-muets (Gand), loi de découverte d'un principe, étalonnage de l'épreuve Borelli-Oléron pour sourds âgés de 4 à 9 ans, évolution spatio-temporelle à partir de deux méthodes d'éducation gestuelle (Louvain, F.).

Louvain, (F.) étudié aussi l'éducation sensori-motrice des handicapés mentaux.

\section{Loisirs}

Sur le plan général, Breuse a entrepris, à partir d'enquêtes, de définir une pédagogie des loisirs (44) (Bruxelles).

On retient deux recherches spécifiques à Louvain (S.P.G.): l'engagement dans les loisirs, mouvements de jeunesse (méthodologie et formation des cadres).

\section{BIBLIOGRAPHIE SOMMAIRE}

1. G. DE LANDSHEERE: Introduction à la vecherche pédagogique, Paris: Armand Colin-Bourrelier - Liège: Thone 1966, 2e éd. - revue et augmentée - Trad. allemande: Einführung in die pödogogische Forschung, Weinheim: J. Beltz Verlag, sous presse.

2. G. DE LANDSheERE: Les tests de connaissances, Bruxelles: Editest 1965.

3. H. Rigaux: Elaboration du test scolaire E.P. 5 - Arithmétique. Bruxelles: Presses Universitaires 1964.

4. P. FRANKARD: Analyse critique de la notion de validité. Paris-Louvain: Nauwelaerts 1958.

5. F. B. Davis: Analyse des items, trad. de R. de Froidmont et H. Willemaers, Paris-Louvain: Nauwelaerts 1966.

6. "L'enseignement secondaire dans la région liégeoise," Bulletin du Grand Liège, no. 49, mars 1963.

7. P. Minon: Facteurs sociaux de la première orientation scolaive, Liège, Travaux de 1'Institut de Sociologie de la Faculté de Droit, 1966.

8. L. Coetsier: "Problèmes gesteld door de beyolking van universitair en middelbaar onderwijs," in Mededelingen van het Laboratorium voor Toegepaste Psychologie en de Dienst voor Studieadvies bij de Rijksuniversiteit te Gent, no. 2.

9. L. COETSIER: "De actuele deelname van beide taalgroepen aan het Belgisch opleidingswezen en de maatschappelijke gevolgen ervan," in Mededelingen $\ldots$, no. 4 .

10. P. Coetsier et A. Bonte: "Topografie van het beschikbaar begaafdheidspotentieel in België en ruimtelijke verschillen in scolarisatiepatroon," in Mededelingen ..., no. 28.

11. S. DE COSTER et al.: "Essais sur la régression sociale virtuelle," Revue de l'Institut de Sociologie, 1963, nos. 1 et 2; 1964, nos. 1 et 2.

12. F. Hotyat: "Les conditions socio-culturelles de la famille et le rendement scolaire au début de l'enseignement post-primaire. In Revue de l'Institut de Sociologie, 1964, no. 2.

13. C. WILLEMART: Etude du vendement pédagogique des classes de neige, Liège: Laboratoire de Pédagogie expérimentale, 1965, à paraître.

14. A. Clausse: Philosophie de l'étude du milieu, Paris: Le Scarabée, 1961.

15. G. DE LANDSheERE: "Pour une pédagogie de la divergence," in Synthèses, 1963, no. 204.

16. A. Bonborr: Etude psychopédagogique de l'arithmétique à l'école primaire, Bruxelles: C.C.U.P. 1962. 
17. A. Bonbolr: Etude psychopédagogique des formes géométriques à l'école primaire. Bruxelles: C.C.U.P. 1962.

18. C.C.U.P.: De deling van gehele getallen en haar moeilijkheden, 1953.

19. J. A. QUaCKELBEEN: Tekenmethodiek op denkpsychologische basis, Tekengids, 1963.

20. M. L. VAN HERREWEGHE: De betekenis van het inzicht bij de muziek-didaktiek .... J. M. Meulenhoff, 1962, $110 \mathrm{pp}$.

21. G. DE LANDSheERE: "Pour une application des tests de lisibilité de Flesch à la langue française," in Le travail Humain, 1-2, Paris, 1963.

22. G. DE LANDSHEERE: "Recherche sur l'évaluation objective des manuels scolaires et des tests," Assoc. Intern. de Péd. expérimentale de Langue française, Acta du XIe Colloque, 1964.

23. G. DE LANDSHEERE: "Lecteurs et lectures: recherches expérimentales sur l'évaluation et le contrôle objectifs," Assoc. Intern. de Péd. expérimentale de Langue française, Acta du XIIe Colloque, 1965.

24. C.C.U.P.: Onderzoek naar de uitspraak van het Nederlands bij leerlingen wit de hoogste klasse van de lagere school in het Kanton Audenaarde-Ronse, Brussel.

25. R. PASQUASY: Contribution à l'étude expérimentale du vocabulaire des élèves de l'enseignement secondaive, Liège: Vaillant-Carmanne 1961.

26. V. D'ESPAllier: Het kind op de drempel van de Lagere School, I Schoolvijpheid, II Leesrijpheid, III Rekenrijpheid, Antwerpen, 1957.

27. L. Coetsier, M. GeEnEns-Thurman en P. Coetsier: Opbouw en Analyse van een Differentiële Geschiktheidsbattery voor het einde van het lager en de Aanvang van het secundair Onderwijs, Deinze: Caecilia Boekhandel, 1964.

28. L. CoetsiER et al.: Analyse van en Predictiemogelijkheden met een Differentiële Geschiktheidsbatterij ..., Deinze: Caecilia Boekhandel 1966.

29. J. Burion: Trois tests de lecture orale, I.S.P.H.; F. Durviaux: Epreuve de compréhension de lecture, I.S.P.H.; L. CLEEMPOEL et al.: La lecture silencieuse au degré supérieur de l'école primaire (exercices étalonnés), I.S.P.H.

30. G. PIRE: Test analytique de français, 11-14 ans, Liège: Dessein.

31. A. Bonboir: Tests de rendement-arithmétique.

32. A. Bonborr: Test pour la mesure de l'acquis arithmétique en fin de scolarité primaire, Liège: Vaillant Carmanne, C.N.R.P.S. 1961.

33. R. Dujardin et L. Adriaknssens: Rekenproef, 4de Jaar, Antwerpen: De Sikkel 1966.

34. WATHelet, Elaboration et exploitation psychopédagogique de deux tests d'acquisitions: Arithmétique-Système métrique, 3 e année primaire, Bruxelles: Editest 1966.

35. G. PIRE: Test d'arithmétique, Liège: Dessain.

36. G. PIRE: Test de latin "Quinquaginta," Liège: Dessain 1963.

37. A. VAN WAYENBERGHE: Essai d'une épreuve objective de connaissances scolaires, Bruxelles: Clérebaut 1956.

38. Arbalestrie: Etude des réserves intellectuelles potentielles de la jeunesse belge, Bruxelles: Centre d'Etude des Problèmes Sociaux 1961.

39. L. DELYS: Inventaire de connaissances acquises en fin d'Humanités, Bruxelles: Clerebaut 1957.

40. B. DE KEGEL en P. COETSiER: "De sociale doeltreffendheid van het studiebeurzenstelsel in het universitair onderwijs," in Mededelingen van het Laboratorium van Toegepaste Psychologie, no. 17, Gand.

41. L. Coetsier en A. Bonte: "Studierendement aan de Universiteit," in Mededelingen .... no. 25.

42. F. Hotyat: Les examens. Les moyens d'évaluation dans l'enseignement, Documents pédagogiques internationaux de l'Institut de l'UNESCO pour l'Education Hambourg. Paris: Bourrelier 1962.

43. F. Hotyat: Les tâches après la classe dans l'enseignement moyen et normal, Bruxelles: Institut de Sociologie 1965.

44. Breuse: Vers une pédagogie des loisirs juvéniles, Bruxelles: Institut de Sociologie de l'U.L.B. 1965.

45. P. Debaty: La mesure des attitudes. Paris: P.U.F. 1967

GilberT de Landsheere, Liège 